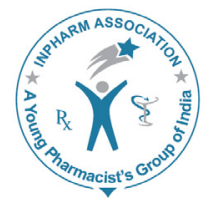

JVP

\title{
Studies on Anti microbial Potential of Non-antibiotics on Resistant Bacteria - A Review
}

\author{
Krishnan Akilandeswari and Kandasamy Ruckmani \\ Department of Pharmaceutical Technology, Bharathidasan Institute of Technology, Anna University, \\ Tiruchirappalli - 620024, India
}

\begin{abstract}
The non-chemotherapeutic compounds, synthetic compounds and naturally isolated compounds possess good antimicrobial activity against microbial infections is a well-known fact. In this state the term non-antibiotics is taken to include a variety of pharmacologically active compounds which are useful in the management of pathological conditions of anon-infectious diseases that modify cell permeability and have been shown to exhibit broad spectrum antimicrobial activity. Of the nonantibiotic medicinal compounds, neuroleptic as well as phenothiazine inhibited the extracellular and intracellular efflux pump system and particularly intracellular efflux pumps has been the main research focus in multidrug resistant gram positive and gram negative microorganisms. The increase and spread of drug resistance is exerted by unsystematic usage of antibiotics for prolonged periods and over use of antimicrobials. Antimicrobial agents are inactivated by the following resistance mechanisms. Multi drug efflux pump and alteration of membrane protein are based on the persistence of resistance genes. A review of literature on a number of research articles suggests that some of these and other membrane active compounds improve the activity of conventional antibiotics, eliminate the natural resistance to particular antibiotics, and exhibit good activity against multi drug resistant form of Mycobacterium tuberculosis and methicillin resistant staphylococcus aureus. Thus, non-antibiotics particularly anti depressants, antipsychotics, anti-hypertensive agent, anti-spasmodic drug, anti-inflammatory agent and Cardio vascular drug have a significant role in the treatment of microbial infections which had shown their synergistic effect with penicillin and some other antibiotics. These effects would help to reduce the dose of antibiotics to patients suffering from specific bacterial infections.
\end{abstract}

Key words: Antimicrobial resistance, anti-hypertensive agent, anti-inflammatory agent, anti-spasmodic drug, Phenothiazine, Mycobacterium tuberculosis.

\begin{tabular}{|c|c|}
\hline \multicolumn{2}{|c|}{ Access this article online } \\
\hline Journal Sponsor & \multirow[b]{2}{*}{$\begin{array}{l}\text { Website: } \\
\text { www.jyoungpharm.org }\end{array}$} \\
\hline \multirow{2}{*}{ www.phcog net } & \\
\hline & DOI: 10.5530/jyp.2015.2.2 \\
\hline
\end{tabular}

\footnotetext{
*Address for correspondence:
}

Dr. K. Ruckmani, Department of Pharmaceutical Technology, Bharathidasan Institute of Technology, Anna University, Tiruchirappalli 620024, India.E-mail: hodpharma@gmail.com other parasites exhibits microbial drug resistance against conventional antibiotics which leads to become a global problem. The excessive use of antibiotics evidenced the development of resistance in microbe's for instance

\section{INTRODUCTION}

Microbial infections include bacterial, viral, fungal, and the development of resistance in microbe's for instance 
vancomycin resistant enterococci (VRE), methicillin resistant staphylococcus aureus (MRSA) and MDR mycobacterium tuberculosis. This problem has been manifested by using the different combinational therapy or new antimicrobial agents to treat drug resistant infections. ${ }^{1}$ In this context, it has become essential to identify effective antimicrobial agents. A different variety of compounds which are employed in the management of pathological conditions of non-infectious diseases have also been shown to alter cell permeability and to exhibit wide range of activity in vitro against specific microorganism. ${ }^{1,2}$ Such compounds are called non-antibiotics. The non-antibiotics express their antimicrobial activity by improvement of antimicrobial activity or by reversal of antibiotic resistance to the original sensitivity of antibiotics as well as by control of efflux pumps. ${ }^{3-5}$ In recent investigations on nonantibiotic compounds such as tricyclic phenothiazine, antiinflammatory agents, cardio vascular drugs, anti-spasmodic agents, anti-hypertensive agents show specific antimicrobial properties when used alone or in conjunction with antibiotics against resistant bacteria. This group of drugs specifically shows their activity through different mechanisms. The first group is having direct antimicrobial activity. The second group can be sub grouped into the helper compounds and the macrophage modulators. The helper compounds alter the permeability of the microorganism and macrophage modulators enhance the bactericidal effect of macrophages that have been phagocytosed by microorganism..$^{6-8}$ Hence, we understand that there is evidence that specific bacterial infections with high risk of resistance to conventional antibiotics can be managed with certain non-antibiotic compound alone or in combination with conventional antibiotics.

\section{The antibiotic and non-antibiotic story}

In medical field, chemotherapy was first developed by Paul Ehrlich in the early part of the $20^{\text {th }}$ century. Such chemotherapeutic agent is more toxic and injurious to the invading organism than to the host. Unexpectedly, studies by Paul Ehrlich (1854-1915) showed that the vital dye methylene blue could be efficacious in the treatment of malaria and the dye trypan red is active against trypanosomiasis in horses and the dye salvarsan had an antisyphilitic action. ${ }^{1,9}$ After the discovery of penicillin by Alexander Fleming, the antimicrobial chemotherapeutic agents usage minimized within a short period of time. A very large number of antibiotic compounds flooded in the medical world and most of these provided quick remedy against gram $(+)$ ve and gram (-) ve microbial infections. The period 1936 to 1962 was considered as golden age of antibiotics. For instance, in 1936 trimethoprim, a sulfa drug, mainly targeted folate metabolism, in 1940, $\beta$-lactam Ampicillin targeted cell wall synthesis, in 1949, chloramphenicol, a phenyl propanoid derivatives and tetracycline, a polyketide drug targeted ribosomes, in 1950 Gentamycin an aminoglycoside targeted ribosomes, in 1952, Erythromycin a macrolide antibiotic targeted ribosomes, in 1958,vancomycin targeted cell wall synthesis, in 1962, ciprofloxacin, a quinolone drug, targeted nucleic acid replication and streptogramins, combination of quinupristin and dalfopristin targeted ribosomes, Following the discovery of ciprofloxacin and streptagamin, next new class of antibiotics, oxazolidinone, was approved almost 40 years laterin 2000, which also targeted ribosomes. Following this period different combinational drugs are most commonly used because they are highly resistance against bacteria's. Augmentin is the combination of amoxicillin \& clavalunate it is used in treatment of infections which produced by $\beta$-lactamase. Cotrimoxazole is the combination of sulfamethoxazole $\&$ trimethoprim it is used in the treatment of urinary tract infection. Rifamycin $\&$ isoniazid is the combination drug used in the treatment of TB. ${ }^{10-11}$ After many years, the unsystematic usage of antibiotics for prolonged periods had its limitations as microorganisms gradually began to rise in the form of antibiotic resistant microbes.

Methodical searching from the mid-1960s, notably by scientists ${ }^{12,13}$ gave real evidences to the fact that many eukaryote-directed drugs have antimicrobial, anti-pathogenic and anti-virulence properties. Such pharmaceutical compounds developed as drugs to treat non-infectious diseases but having antimicrobial activities are called non-antibiotics. ${ }^{14}$ In pharmacology, drugs are catalogued as antimicrobial agents or drugs for noninfectious diseases. ${ }^{15}$ Searching amongst non-antibiotics brought to light that they covered a wide spectrum, including antihistamines such as bromodiphenhydramine, diphenhydramine, methdilazine, promethazine and trimeprazine, Escitalopram oxalate ${ }^{16}$ antihypertensives such as Amlodipine, ${ }^{17}$ Dobutamine, ${ }^{18}$ Lacidinpine, ${ }^{19}$ Nifidipine ${ }^{20}$ antipsychotics such as chlorpromazine, flupenthixol, fluphenazine, prochlorperazine, thioridazine (THZ), ${ }^{21,23}$ trifluoperazine and triflupromazine, tranquilisers such as promazine. The above non antibiotics can effectively manage the microbial infections of many viruses, mycoplasma, gram-negative and gram-positive bacteria, mycobacterium, yeasts, protozoa, plasmodia, leishmania, trypanosomes, and helminthes. ${ }^{912,13}$ Many of these are capable of modifying cellular permeability, inhibiting efflux pumps, synergize with various antibiotics, especially for reversal of antibiotic resistance and thus acting as helper compounds. Prontosil, a sulfonamide group exhibits their additional antimicrobial 
activity. Similarly a synthetic compound nitrofurantoin selectively inhibits the bacteria invading the urinary tract but can also damage the mammalian DNA. The phenazine dye clofazemine possesses anti-histaminic, anti-inflammatory as well as powerful antibacterial actions against leprosy bacilli by inhibiting DNA template formation. Moreover the analgesic aspirin (acetyl salicylic acid) exhibits their additional function and is now prescribed to act against thrombosis and also act against microbial infections. While cyproheptadine is an anti-histamine and is also an anabolic stimulant. ${ }^{1}$ It is reported that post cataract inflammation could be managed with diclofenac- gentamycin eye drops which was proved to be more effective than gentamycin eye drops. ${ }^{24}$ Ascorbic acid also enhances the antibacterial activity with aminopenicillinampicillin. ${ }^{25,26}$ Thioridazine could be used as a potent anti-tubercular drug against multidrug-resistant (MDR) microorganisms. ${ }^{21}$

\section{DISCUSSION}

\section{Antimicrobial activity of anti-hypertensive agent -methyl -L-DOPA}

Previous in vitro studies reported that $\mathrm{m}-\mathrm{L}-\mathrm{DOPA}$ was found to possess antibacterial activity against gram positive and gram negative microorganisms. The Minimum Inhibitory Concentration of m-L-DOPA ranged from $10-200 \mu \mathrm{g} / \mathrm{ml}$ when challenged with virulent bacterium. The MIC of m-L-DOPA was much higher than the MIC of conventional antibiotics. The susceptible strains such as Mycobacterium tuberculosis H37Rv102 were inhibited at lower dose of conventional anti tubercular agents ( 0.5 to $2 \mu \mathrm{g} / \mathrm{ml}$ ), whereas poly and multidrug resistant clinical isolates were inhibited at much higher concentration and some of the strains exhibited their resistance even at higher concentration. The MIC of Methyl L-DOPA against the same strain was $15 \mu \mathrm{g} / \mathrm{ml}$, while $25 \mu \mathrm{g} / \mathrm{ml}$ concentrations were sufficient for the drug resistant strain. Previous in vivo studies observed that M.tuberculosis H37RV102 infected mice were seen with several tubercles in the liver, spleen, lungs, peritoneum and intestine. After administration of $\mathrm{m}$-L-DOPA there was minute tubercle present in liver, spleen and peritoneum but no tubercle was detected in the lungs. From these observations, m-L-DOPA could be used as a starting material for further new and safe drugs with enhanced anti mycobacterium activity. ${ }^{27}$

\section{Anti-microbial activity of anti-spasmodic drug- Dicyclomine}

Dicyclomine is an ant cholinergic and antispasmodic drug, having significant antimicrobial activity against gram positive and gram negative microorganism. Of the 414 bacterial isolates tested with dicyclomine, the observed MIC was $5-25 \mu \mathrm{g} / \mathrm{ml}$ concentrations. The duration of action of dicyclomine in human body was found to be approximately 5-6 hrs. No mortality was recorded in the two groups of mice injected with 30 and $70 \mu \mathrm{g} / \mathrm{ml}$ of dicyclomine throughout the observation period for a maximum of $100 \mathrm{hrs}$. In vivo protection of mice was observed from the number of viable bacteria in heart blood, liver and spleen of mice. Intra peritoneal administration of dicyclomine significantly reduced the number of viable bacteria. This drug is satisfactory in human toxicity tests and it is already in regular therapeutic usage which is being developed as second or first line of drug in many bacterial infections especially gastrointestinal tract infections. The efficacy is enhanced by structural modification and synergistic combination of drug with conventional antibiotics. From the earlier reports phenothiazine which contain tricyclic benzene rings possess moderate to powerful antimicrobial action. In dicyclomine, the presence of two cyclic rings and nitrogen in the secondary tertiary state may be playing a key role in conferring antimicrobial activity to this compound. ${ }^{28,29}$

\section{Anti-microbial potentiality of Cardio vascular drug- oxyfedrine}

The anti anginal and coronary vasodilator cardiovascular drug oxyfedrine hydrochloride is reported to possess powerful antibacterial activity both in vitro and in vivo in mouse experiments. From the previous studies ten CVS drugs were preliminary screened for antibacterial activity by testing them in vitro against eight sensitive bacteria. All the bacteria were resistant to clonidine, digoxin, dipyridamole, nitrendipine and lacidipine enlapril and verapamil inhibit their activity against few bacteria at high concentration (400-800 $\mu \mathrm{g} / \mathrm{ml})$. Dobutamine and oxyfedrine showed significant antibacterial activity against most of the bacteria. The highly virulent bacterium when administered with drug significantly reduced the bacterial growth. Oxyfedrine can be administered to a human being at the dose of 25-100 $\mu \mathrm{g} / \mathrm{ml}$ orally. Activity of the drug takes place at the end of $30 \mathrm{~min}$ to $2 \mathrm{hrs}$. In vivo animal studies showed significant protection of mouse with $30 \mu \mathrm{g} / \mathrm{ml}$ of drug and no toxicity was observed. Even at a higher concentration of $60 \mu \mathrm{g} /$ $\mathrm{ml}$ of drug, it gave better protection of mouse. Thus it was concluded that in vivo protection test confirmed the activity of oxyfedrine against most viable bacteria in heart, blood, liver, and spleen of mice. The above study suggests that oxyfedrine has a potential for being developed into a powerful antimicrobial agent, and its efficacy can be enhanced by further altering the structure and synergize 
with other antibiotics. ${ }^{30,31}$

\section{In vitro and in vivo synergistic effect of anti-inflammatory agent-Diclofenac against Listeria monocytogenes}

One of the most widely studied non-antibiotics is the non-steroidal anti-inflammatory drug diclofenac sodium. It has been reported that this agent possess in vitro and in vivo antimicrobial activity against a variety of microorganism such as gram positive and gram negative, as well as mycobacterium. Diclofenac synergistically enhances the efficacy of the antibiotic streptomycin and even other non-antibiotics like trifluoperazine against specific bacteria Listeria monocytogenes which is used as a food born microorganism. It has been on the record that diclofenac at $2.5 \mu \mathrm{g} / \mathrm{ml}$, administered orally to female mice against Listeria monocytogenes significantly reduced bacterial counts in liver, spleen, decreased hepatic colonization, necrosis and caused up regulation of expression of inflammatory cytokines (IFN) compared to drug free control. The synergistic response of such activity in combination indicate that these non-antibiotic will eventually develop in the creation of a new array of promising adjuvants to existing therapeutics used for the treatment of problematic microbial infections like listeriosis. ${ }^{32,33}$ One more non antibiotics non-steroidal anti-inflammatory drug Nimesulide also used the treatment of antibacterial activity against Staphylococcus aureus NCIM 2079, Gram- negative organisms such as Pseudomonas aeruginosa NCIM 2036, Klebsiella pneumonia NCIM 2719, Enterobacter cloacae NCIM 2164, Proteus mirabilis, Pseudomonas fluoroceae. ${ }^{34}$

\section{Helper compound enhancing antimicrobial effect- Azelastine}

Previous in vitro studies suggest that azelastine is a phthalazinone non-sedating new generation antihistaminic drug widely used in the management of allergic condition. Azelastine possess certain antibacterial activity against a variety of microorganisms due to its ability to induce alterations in membrane permeability and disruption of its integrity. The effect of azelastine in the antibacterial activity of antibiotics was tested by MIC of antibiotics in the presence and absence of antibiotics. The MIC of azelastine was $125-250 \mu \mathrm{g} / \mathrm{ml}$ whereas combined with antibiotics the MIC was reduced fourfold or more..$^{35}$

\section{Mechanism of anti-microbial activity of phenothiazine}

Microbial system that contains different types of transporters are Major Facilitator Super family (MFS), Resistance nodulation division (RND), Small multidrug resistance (SMR) and multi drug resistance (MDR), especially MDR type transporter is one of the major intracellular efflux pump system in mycobacteria. That efflux mechanism identifies the noxious agents such as antimicrobial agents, detergents (that act on the cell envelope) and extrude from the cell envelope. When these pumps are over expressed the bacterium becomes resistant to a variety of unrelated antibiotics, hence their MDR phenotype appeared. Due to over expression of efflux pump on the cause of ATP dependent enzyme system are generated by proton motive force which gives the energy.

Phenothiazines have been shown to inhibit in vitro growth of Multi Drug Resistance (resistance to rifampin and isoniazid) Mycobacterium tuberculosis. The killing of intracellular bacteria promoted by phenothiazine can inhibit many transport processes potassium and calcium pump activation. The mechanism of action of phenothiazine inhibits the transport of calcium $\left(\mathrm{ca}^{2+}\right)$ by preventing its binding with $\mathrm{ca}^{2+}$ proteins such as calmodulin. This means that enzyme system which is dependent upon $\mathrm{ca}^{2+}$ that is responsible for the generation of cellular energy from hydrolysis of ATP, ATP dependent $\mathrm{ca}^{2+}$ is inhibited there by energy giving mechanism is cut off. Then the phenothiazine reverses the MDR phenotype of the bacterium and render susceptible to those antibiotics to which they were initially resistant. Increased permeability ensures phenothiazine molecules reach the DNA, intercalate between the bases, inhibit the entire DNA and hence inhibit transcription and translation processes. Phenothiazine has been considered as an adjuvant for the treatment of Mycobacterium tuberculosis. ${ }^{36-49}$

Many phenothiazines chlorpromazine, promazine, promethazine, prochlorperazine, methdilazine, fluphenazine, trimeprazine, trifluoperazine, triflupromazine, thioridazine, flupenthixol have shown their anti-microbial effect and some of these compound exhibits synergistic activity with antibiotics against microbial infections. Trimeprazine is synergistic with trimethoprim. Flufenazine and chlorpromazine have shown their synergistic effect with penicillin and some other antibiotics. These effects would help to reduce the dose of antibiotics to patients suffering from specific bacterial infections. Moreover thioridazine not only exhibits antimicrobial action and also has an ability to kill drug resistant plasmid DNA in bacterial infections. ${ }^{45}$ The in vitro activity of phenothiazine that inhibit the microbial growth was many hundred fold higher at lower concentration, but the same effect was not attained when it was tried with infected patients and have concluded that the in vivo efficacy of the phenothiazine is not related to that produced in vitro. ${ }^{\text {? }}$ 


\section{CONCLUSION}

The group of compounds termed as non-antibiotics exhibit their direct antimicrobial activity against microbial resistance especially MDR-TB microbial infection. Moreover it acts as a helper compound by enhancing the activity of conventional antibiotics and their ability in specific instances to reverse resistance of conventional antibiotics to original sensitivity. Many phenothiazines derivatives have shown synergistic activity with several antibiotics thereby lowering the doses of antibiotics administered to patients suffering from specific bacterial infections. The major difficulties in the continued use of chlorpromazine for the treatment of psychosis lead to give a large number of side effects. Yet thioridazine

\section{REFERENCES}

1. Mazumdar K, Kumar KA, Dutta NK. Potential role of the cardiovascular non-antibiotic (helper compound) amlodipine in the treatment of microbial infections: scope and hope for the future. Int. J. Antimicrob. Agents 2010; 36(4): 295-302.

2. Coutinho HDM, Matias EFF, Santos KKA, Tintino SR, Souza CES, et al. Enhancement of the Norfloxacin Antibiotic Activity by Gaseous Contact with the Essential Oil of Croton zehntneri. J Young Pharm 2010; 2(4): 362-4.

3. Kristiansen JE, Hendricks O, Delvin T, Butterworth TS, Aagaard L, Christensen JB. Reversal of resistance in microorganisms by help of non-antibiotics. J Antimicrob Chemother. 2007; 59(6): 1271-9.

4. Molnar J. The reversal of drug resistance from bacteria to cancer cells. Curr. Drug. Targets 2006; 7(7): 789-91.

5. Viveiros M, Jesus A, Brito A, Leandro C, Martins M, Ordway D, et al. Inducement and reversal of tetracycline resistance in Escherichia coli $\mathrm{K}-12$ and expression of proton gradient-dependent multidrug efflux pump genes. Antimicrob. Agents. Chemother. 2005; 49(8): 3578-82.

6. Kaatz GW, Moudgal VV, Seo SM, Kristiansen JE. Phenothiazines and thioxanthenes inhibit multidrug efflux pump activity in Staphylococcus aureus. Antimicrob. Agents Chemother. 2003; 47(2): 719-26.

7. Amaral L, Lorian V. Effects of chlorpromazine on the cell envelope proteins of Escherichia coli. Antimicrob. Agents. Chemother. 1991; 35(9): 1923-4.

8. Viveiros M, Amaral L. Enhancement of antibiotic activity against poly-drug resistant Mycobacterium tuberculosis by phenothiazines. Int. J. Antimicrob. Agents. 2001; 17(3): 225-8.

9. Martins M, Dastidar SG, Fanning S, Kristiansen JE, Molnar $\mathrm{J}$, Pages JM, et al. Potential role of non-antibiotics (helper compounds) in the treatment of multidrug-resistant Gram-negative infections: mechanisms for their direct and indirect activities. Agents. Int. J. Antimicrob. 2008; 31(3): 198-208.

10. Christopher Walsh. Where will new antibiotics come from? Nature reviews 2003; 1(1): 65 .

11. Saswati S, Madhab KC. Antibiotic Resistance of Bacteria: A Global Challenge. Resonance 2012; 17(2): 177-91.

12. Dutta NK, Annadurai S, Mazumdar K, Dastidar SG, Kristiansen $\mathrm{JE}$, Molnar J, et al. Potential management of resistant microbial infections with a novel non antibiotic: the anti-inflammatory drug diclofenac sodium. Agents. Int. J. Antimicrob. 2007; 30(3): 242-9.

13. Mazumdar K, Dastidar SG, Park JH, Dutta NK. The antiinflammatory non antibiotic helper compound diclofenac: an antibacterial drug target. Eur. Clin. Microbiol. Infect. Dis. 2009; is a derivative of $\mathrm{CPZ}$ that is effective even at lowest concentration than $\mathrm{CPZ}$ but producing major side effects somnia. Because of these facts these discovery routes have not yet entered into clinical trials.

\section{CONFLICT OF INTEREST}

Authors are declared there is no conflict of interest.

\section{ACKNOWLEDGEMENT}

The authors would like to thank all the authors of the research articles which were of great help in writing this review.

28(8): 881-91.

14. Kristiansen JE. Antimicrobial activity of non-antibiotics. ASM News 1991; 57: 135-9.

15. Williams JD. The Garrod Lecture. Selective toxicity and concordant pharmacodynamics of antibiotics and other drugs. J. Antimicrob. Chemother. 1995; 35(6): 721-37.

16. Akilandeswari K, Ruckmani K, Ranjithmathan V. Efficacy of Antibacterial Activity of Antibiotics Improved with Anti-Depressant Drug Escitalopram. Int. J. Pharm. Sci. Rev. Res. 2013; 21(2): 14, 71-4.

17. Kumar KA, Ganguly, Mazumdar K, Dutta NK, Dastidar SG, Chakrabarty AN. Amlodipine: a cardiovascular drug with powerful antimicrobial property. Acta. Microbiol. Pol. 2003; 52(3): 285-92.

18. Sarkar A, Kumar KA, Dutta NK, Chakraborty P, Dastidar SG. Evaluation of in vitro and in vivo antibacterial activity of dobutamine hydrochloride. Indian. J. Med. Microbiol. 2003; 21(3): 172-8.

19. Dasgupta A, Jeyaseeli L, Dutta NK, Mazumdar K, Karak P, Dastidar SG, et al. Studies on the antimicrobial potential of the cardiovascular drug lacidipine. In vivo. 2007; 21(5): 847-50.

20. Pal T, Dutta NK, Mazumdar K, Dasgupta A, Jeyaseeli L, Dastidar SG. Assessmentof antibacterial activity of the cardiovascular drug Nifedipine. Oriental. Pharm. Exp. Med. 2006; 6(2): 126-33.

21. Dasgupta A, Mukherjee S, Chaki S, Dastidar SG, Hendricks O, Christensen JB, et al. Thioridazine protects the mouse from a virulent infection by Salmonella enteric serovar Typhimurium 74 . Agents. Int. J. Antimicrob. 2010; 35(2): 174-6.

22. Dutta NK, Mehra S, Kaushal D. A mycobacterium tuberculosis sigma factor network responds to cell-envelope damage by the promising anti-mycobacterial thioridazine. PLOS One. 2010; 5(4): 10069 e.

23. Amaral L, Boeree MJ, Gillespie SH, Udwadia ZF, Van Soolingen D. Thioridazine cures extensively drug-resistant tuberculosis (XDRTB) and the need for global trials is now. Agents. Int. J. Antimicrob. 2010; 35(6): 524-6.

24. Shepherd WF, Fsadni MG, Raj SA. A clinical evaluation of diclofenac-gentamicin combination eye drops in the control of inflammation after cataract surgery. Diclofenac-Gentamicin versus Gentamicin Study Group. Ocul. Immunol. Inflamm. 1998; 6(1): 13-8.

25. Akilandeswari K. Estimation of antimicrobial activity of ascorbic acid in superoxide dismutase level with amino penicillin ampicillin Int. J. Pharm. Sci. Rev. Res. 2013; 20(2): 167-9.

26. Akilandeswari K, Vijaya C, Babu Dhandabani. An in vitro investigation study on the interaction between certain dietary anti-oxidants with ampicillin. The antiseptic 2007; 104(12): 615-8. 
27. Dutta NK, Mazumdar K, Dastidar SG, Chakrabarty AN, Shirataki $\mathrm{Y}$, Motohashi N. In vitro and in vivo antimycobacterial activity of an antihypertensive agent methyl-I-DOPA. In Vivo. 2005; 19(3): 539-45.

28. Karak P, Kumar KA, Mazumdar K, Mookerjee M, Dastidar SG. Antibacterial potential of an antispasmodic drug dicyclomine hydrochloride. Indian. J. Med. Res. 2003; 118: 192-6.

29. Karaka P, Kumar KA, Basu LR, Dasgupta A, Ray R, Dastidar SG. Experimental analysis of antimicrobialaction of Dicyclomine Hydrochloride. Biol. Pharm. Bull. 2004; 27(12): 2010-3.

30. Mazumdar K, Ganguly K, Kumar KA, Dutta NK, Chakrabarty AN, Dastidar SG. Antimicrobial potentiality of a new non-antibiotic: the cardiovascular drug oxyfedrine hydrochloride. Microbiol. Res. 2003; 158(3): 259-64.

31. Mazumdar K, Dutta NK, Kumar KA, Dastidar SG. In vitro and in vivo synergism between tetracycline and the cardiovascular agent Oxyfedrine $\mathrm{HCl}$ against common bacterial strains. Biol. Pharm. Bull. 2005; 28(4): 713-7.

32. Dutta NK, Mazumdar K, Baek MW, Kim DJ, Na YR, Park SH, et al. In vitro efficacy of diclofenac against Listeria monocytogenes. Eur. J. Clin. Microbiol. Infect. Dis. 2008; 27(4): 315-9.

33. Dutta NK, Mazumdar K, Seok SH, Park JH. The anti-inflammatory drug diclofenac retains anti-listerial activity in vivo. Lett. Appl. Microbiol. 2008; 47(2): 106-11.

34. Akilandeswari K, Ruckmani K, Abirami S. Enhancement of the efficacy of antibiotics ciprofloxacin and gentamycin against gram positive and gram negative micro organism with non antibiotic Nimesulide. Int. J. pharm. pharmsci. 2013: 5(3): 627- 30.

35. El-Nakeeb MA, Abou-Shleib HM, Khalil AM, Omar HG, El-Halfawy OM. Reversal of antibiotic resistance in Gram-positive bacteria by the antihistaminic azelastine. APMIS. 2012; 120(3): 215-2.

36. Kaatz GW, Moudgal VV, Seo SM, Kristiansen JE. Phenothiazines and thioxanthenes inhibit multidrug efflux pump activity in Staphylococcus aureus. Agents. Antimicrob. Chemother. 2003; 47(2): 719-26.

37. Nacsa J, Nagy L, Sharples D, Hevér A, Szabo D, Ocsovszki I, et al. The inhibition ofSOS-responses and MDR by phenothiazine-metal complexes. Anticancer Res. 1998; 18(4C): 3093-8.

38. Molnar J, Hever A, Fakla I, Fischer J, Ocsovski I, Aszalos A, et al. Inhibition of the transport function of membrane proteins by some substituted phenothiazines in E. coli and multidrug resistant tumor cells. Anticancer. Res. 1997; 17(1A): 481-6.

39. Viveiros $M$, Leandro $C$, Amaral L. Mycobacterial efflux pumps and chemotherapeutic implications. Agents. Int. J. Antimicrob. 2003; 22(3): 274-8.

40. Viveiros M, Portugal I, Bettencourt R, Victor TC, Jordaan AM, Leandro $C$, et al. Isoniazid-induced transient high-level resistance in Mycobacterium tuberculosis. Agents. Antimicrob. Chemother. 2002; 46(9): 2804-10.

41. Kristiansen MM, Leandro $C$, Ordway $D$, Viveiros $M$, Pacheco $T$, Kristiansen JE, et al. Phenothiazine salter resistance of methicillinresistant strains of Staphylococcu aureus (MRSA) to oxacillin in vitro. Agents. Int. J. Antimicrob. 2003; 22(3): 250-3.

42. Kristiansen $M M$, Leandro $C$, Ordway $D$, Martins $M$, Viveiros $M$, Pacheco T, et al. Thioridazine reduces resistance of methicillinresistant Staphylococcus aureus by inhibiting a reserpine-sensitive efflux pump. In vivo. 2006; 20(3): 361-6.

43. Amaral L, Molnar J. Why and how the old neuroleptic thioridazine cures the XDR-TB patient. Pharmaceuticals 2012; 5(9): 1021-31.

44. Amaral L,Udwadia Z, Abbate E, van Soolingen $D$. The added effect of Thioridazine in treatment of resistant TB. Int. J. Tuberc. Lung Dis. 2012; 16(12): 1706-8.

45. Dastidar SG, Kristiansen JE, Molnar J, Amaral L. Role of phenothiazines and structurally similar compounds of plant origin in the fight against infections by drug resistant bacteria. Antibiotics 2013; 2(1): 58-72.

46. Amaral L, Martin M, Viveiros M. Enhanced killing of intracellular multidrug-resistant Mycobacterium tuberculosis by compounds that affect the activity of efflux pumps. J. Antimicrob. Chemother 2007; 59(6): 1237-46.

47. Machado D.Couto I, Perdigao J, Rodrigues L, Portugal I, et al. Contribution of efflux to the emergence of isoniazid and multidrug resistance in Mycobacterium tuberculosis. PLoS One. 2012; 7(4): e34538.

48. Rodrigues L, Machado D, Couto I, Amaral L, Viveiros M. Contribution of efflux activity to isoniazid resistance in the Mycobacterium tuberculosis complex. Infect. Genet. Evol. 2012; 12(4): 695-700.

49. Amaral L, Fanning S, Pagès JM. Efflux pumps of gram-negative bacteria: Genetic responses to stress and the modulation of their activity by $\mathrm{pH}$, inhibitors, and phenothiazines. Adv. Enzymol. Relat. Areas Mol. Biol. 2011; 77 61-108. 